\title{
LVIII. The curvature method of determining the surface-tension of liquids
}

\section{C.V. Raman M.A.}

To cite this article: C.V. Raman M.A. (1907) LVIII. The curvature method of determining the surface-tension of liquids, Philosophical Magazine Series 6, 14:83, 591-596, DOI: 10.1080/14786440709463720

To link to this article: http://dx.doi.org/10.1080/14786440709463720

曲 Published online: 16 Apr 2009.

Submit your article to this journal $₫$

Џll Article views: 6

Q View related articles $₫$ 
Method of determining Surface-Tension of. Liquids. $\quad 591$ where $\mathrm{K}$ and $\epsilon$ are arbitrary constants to be chosen to satisfy the initial conditions. Thus the segments of the bar are like those of a string fixed at the ends. The frequencies of the possible tones are, however, quite different. For, in this case of the bar the frequencies are proportional to $n^{2}$ instead of to $n$ simply as for a string. Thus the frequency of the $n$th tone is given by

$$
\mathrm{N}_{n}=\frac{\kappa b n^{2} \pi}{2 l^{2}}=n^{2} \mathrm{~N}_{1} \ldots . . . .
$$

University College, Nottingham.

May 30, 1907 .

LVIII. The Curvature Method of determining the SurfaceTension of Liquids. By (Y. V. Raman, M.A.*

[Plate XV.]

T ORD KELVIN, in his lecture on Capillarity (published 1 in his 'Popular Lectures and Addresses'), describes this method as a practicable one for measuring the surfacetension of liquids. In the following account is described an arrangement by which this method is rendered a convenient and fairly accurate one, and the results of a series of determinations made by the method are tabulated.

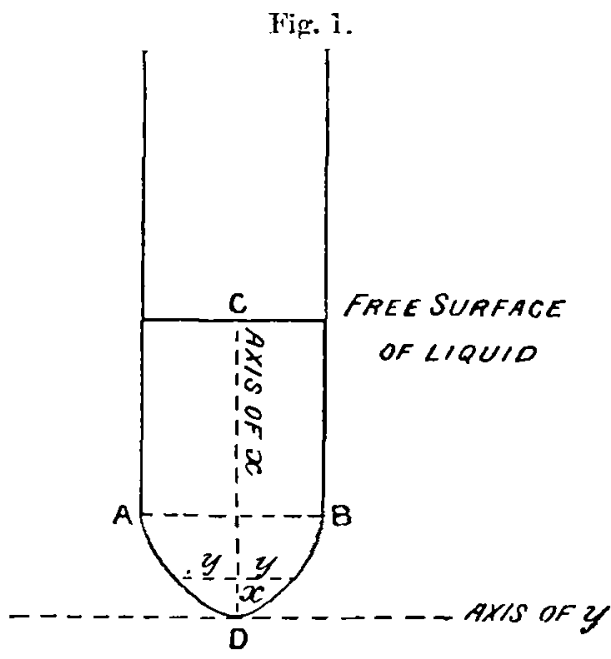

The Theory of the Method.

Let ADB be the principal section of a drop hanging down from a tube, and $\rho$ the radius of curvature of $A \overline{D B}$ at the

* Communicated by the Author.

2 ll 2 
point $D$. If $T$ is the surface-tension, then

$$
\frac{2 \mathrm{~T}}{\rho}=g \sigma \cdot \mathrm{CD}
$$

$\sigma$ being the density of the liquid, since CD is the head at the point $D$. This is true, provided that the surface of the drop is one of revolution about (DD. $\rho$ can be determined by measuring the coordinates $x$ and $y$, of points on the curve ADB.

$$
\rho=\operatorname{I}_{x=0} \frac{y^{2}}{2 x} \text {. }
$$

In practice, it is quite sufficient to make the measurements of $x$ and $y$ close to the vertex for two or more values of $x$. The value of the ratio $\frac{y^{2}}{2 x}$ at different points close to the vertex varies only very slightly, and any variation shown can be corrected for, by calculating the value of the ratio for the point $x=0$ from the observed values of the ratio. The reason why the ratio $\frac{y^{2}}{2 x}$ varies very little can easily be seen. As we go up along the surface from the vertex $D$ the head diminishes, and therefore also the sum. of the principal curvatures. The section of the surface is therefore nearer being a parabola than a circle, for the sum of the principal curvatures of a paraboloid of revolution diminishes as we recede from the vertex.

The measurements of $x$ and $y$ were not made on the drop, but, adopting a more accurate and convenient method, on a photograph of the surface. The apparatus designed for the purpose is shown in fig. 2.

A tube 2 cms. diameter is connected up with a tube $6 \mathrm{mms}$. diameter by caoutchouc tubing. The arrangement shown in the figure is filled up with the liquid. The small tube can easily be adjusted

Fig. 2.

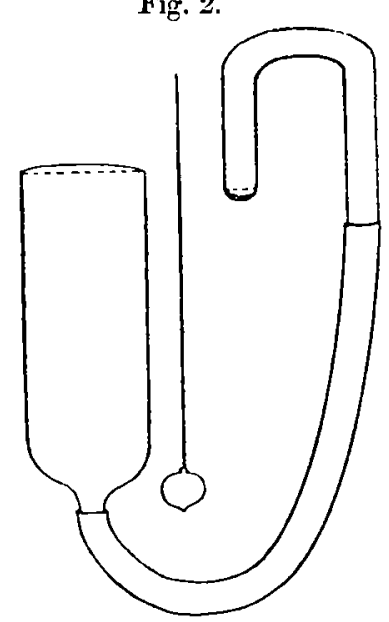
so that the liquid bulges out of both tubes, the concavity being in opposite directions in the two. A plumb-line 
hanging between the two gives the direction of the vertical.: The apparatus is then photographed in the following manner :-

A horizontal beam of parallel light is produced by an electric spark placed at the focus of an achromatic lens of long focal length (about 1.5 metres), and is thrown on the apparatus. The shadow cast by the tubes is then photographed on a plate held vertically as near to them as possible.

The negative is then measured under a sliding microscope. It is coupled, film to film, with a "réseau" plate on which is ruled a set of squares (side $=0.5027 \mathrm{~cm}$.), and placed on an inclined plane so that it has freedom of up and down movement. The microscope, which is capable of motion sideways, has two scales at right angles to each other in its focal plane. The glass plate on which these scales are ruled can be moved in the focal plane by a micrometer-screw. The réseau plate is first adjusted so that the rulings on it are parallel and perpendicular to the shadow of the plumb-line on the plate. By rotating the eyepiece and the photographic plate on the stage, the scales in the focal plane are adjusted parallel to the rules on the réseau plate and to the lines of travel of the microscope and the photographic plate.

The objective of the microscope (which is a small one) produces very little magnification, but the eyepiece is a rairly powerful one. The scales in the focal plane (1 division $=\frac{1}{10} \mathrm{~mm}$.) serve only as auxiliaries, for the readings made on them can easily be expressed in terms of the side of the squares on the réseau plate, the absolute value of which is known. The $x$ and $y$ coordinates of any point on the meniscus can thus be measured and reduced to centimetres. What was directly read off was not the $y$ coordinate but $2 y$. From these measurements the radius of curvature at the vertex of the curve can be deduced in the manner mentioned above. If the variation of $\frac{y^{2}}{2 x}$ is not negligible, then $x$ is
written equal to

$$
a y^{2}+b y^{4}
$$

since the curve is symmetrical about the axis of $x$. From the observational equations

$$
x_{1}=a y_{1}^{2}+b y_{1}^{4}, \& \mathrm{c} \text {. }
$$

two normal equations for the constants $a$ and $b$ can be 
deduced and $a$ determined ; and

$$
a=\frac{1}{2 p} .
$$

Further, by means of the ruling on the reseau plate, the difference of level between the vertices of the two curves can be measured. This gives the pressure corresponding to the observed curvature.

In practice, it was found that the curvature of the larger meniscus could not altogether be neglected. It amounted to from 2 to 4 per cent. of the curvature of the smaller. This could be measured and allowed for. If $\frac{1}{\rho}$ is the curvature of the smaller and $\frac{1}{\mathbf{R}}$ that of the larger meniscus,
then

$$
2 \mathrm{~T}\left(\frac{1}{\rho}-\frac{1}{\mathrm{R}}\right)=g \sigma h,
$$

$h$ being the difference of level between the vertices.

The liquid used in the experiments was clean distilled water. Precautions were taken that in transferring the liquid into the apparatus no contamination occurred. That the surface whose tension was measured was free from greasy contamination was ensured by allowing the water to itun down from the small tube for some time. The surface of the drop that was finally left hanging was, therefore, an absolutely fresh one, and any oily film originally on the surface would have been reduced in thickness to a very great extent. The parallelism of the beam used to photograph the drop was tested by a telescope adjusted for infinity. To have the photograph as sharp as possible, the plate was held only $3 \mathrm{cms}$. behind the apparatus. It was found that it was necessary to adjust the wide tube so that it might be approximately vertical. If not, there was reason to think that the two principal curvatures at the highest point of the large meniscus would not be equal. This was really the weakest point in the whole work. The writer hopes to repeat the work with a tube so wide that the curvature of the meniscus above it can entirely be neglected.

As an illustration of the method, I give a table of the measurements made on one of the plates (see PI. XV.). 
of determining the Surface-Tension of Liquids. $\quad 595$

Difference of level between the vertices

$=44.37$ divisions of the microscope scale;

$=0.2826 \mathrm{~cm}$.

\begin{tabular}{|c|c|c|c|}
\hline $\begin{array}{c}\text { Difference } \\
\text { of level. }\end{array}$ & $x$. & $2 y$. & $\frac{y^{2}}{2 x} \cdot$ \\
\hline & $3 \cdot 00$ & $45 \cdot 80$ & $87 \cdot 06$ \\
& 4.00 & $52 \cdot 78$ & $87 \cdot 05$ \\
$44 \cdot 37$ & $5 \cdot 00$ & 58.92 & 86.79 \\
& 600 & $64 \cdot 71$ & 87.24 \\
\hline & & Mean ....... & 87.03 \\
\hline
\end{tabular}
From this it appears that for this plate $\frac{y^{2}}{2 x}$ is very nearly
indeed a constant $=87.03$ divisions.

Of course, the edge of the shadow was not a sharp line but a series of very narrow diffraction-fringes. In measuring the difference of level, the position of the geometrical edge of the shadow ( $G$ in fig. 3) was estimated and used. But in making the measurement of curvature, a better-defined point ( $B$ in fig. 3) in the curve of illumination was used.

Fig. 3.
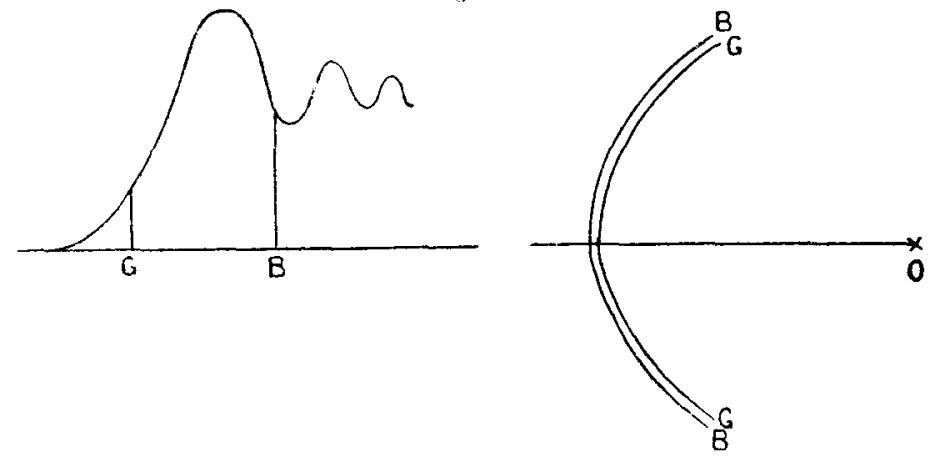

GB was carefully measured and found to be $1 \cdot 16$ divisions.

$$
\rho=87 \cdot 03-1 \cdot 16=85.87 \text { divisions. }
$$

$\mathbf{R}$, by measurement (in a similar fashion) was found to be 3400 divisions. 
The surface-tension calculated from these measurements was (temp. $=30^{\circ} \mathrm{C}$.) found to be $77 \cdot 0$ dynes per $\mathrm{cm}$.

Another plate, not so sharp, gave $76 \cdot 3$ dynes per $\mathrm{cm}$.

A third, in taking which the wide tube happened to be out of the vertical, gave no definite result on account of the uncertainty of the value of the curvature of the large meniscus. The value of the surface-tension was, however, estimated to lie between 75 and 77 dynes per $\mathrm{cm}$.

The accepted value for the surface-tension of clean distilled water at $30^{\circ} \mathrm{C} .=71 \cdot 3$ dynes per cm. (T. P. Hall, Phil. Mag. 1893). The method described in this article gives results which are fairly self-consistent. The cause of the difference has as yet not been found and is under investigation.

The experiments and observations recorded in this note were made at the Physical Laboratory of the Presidency College, Madras.

IIX. On the Relation of the Sensitiveness of the Ear to Pitch, investigated by a new Method. By Lord RAYLEIGH, O.M., P.R.S.*

T $N$ a former research $+\mathrm{I}$ examined the sensitiveness of the ear to sounds of different pitch with results which were thos summarized :-

$$
\begin{array}{rrrr}
e^{\prime}, & \text { frequency } & =256, & s=6.0 \times 10^{-9}, \\
a^{\prime}, & \quad & =384, & s=4.6 \times 10^{-4}, \\
c^{\prime \prime}, & , \quad & =512, & s=4.6 \times 10^{-9},
\end{array}
$$

no reliable distinction appearing between the two last numbers. "Even the distinction between $6 \cdot 0$ and $4 \cdot 6$ should be accepted with reserve; so that the comparison must not be taken to prove much more than that the condensation necessary for audibility varies but slowly in the singly dashed octave." Here $s$ denotes the condensation (or rarefaction) which in one respect is a maximum and in another a minimum. It is the maximum condensation which occurs during the course of the vibration, but the vibration (and $s$ with it) is the minimum capable of impressing the ear in a progressive wave. The method employed depended upon a knowledge of the rate at which energy was emitted from a resonator under excitation by a freely vibrating tuning-

* Communicated by the Author.

† Phil. Mag. xxxviii. p. 365, 1894: Scientific Papers, iv. p. 125. 
RAMAN.

Phil, Mas. Ser. 6, Vol. 14. Pl. XV.

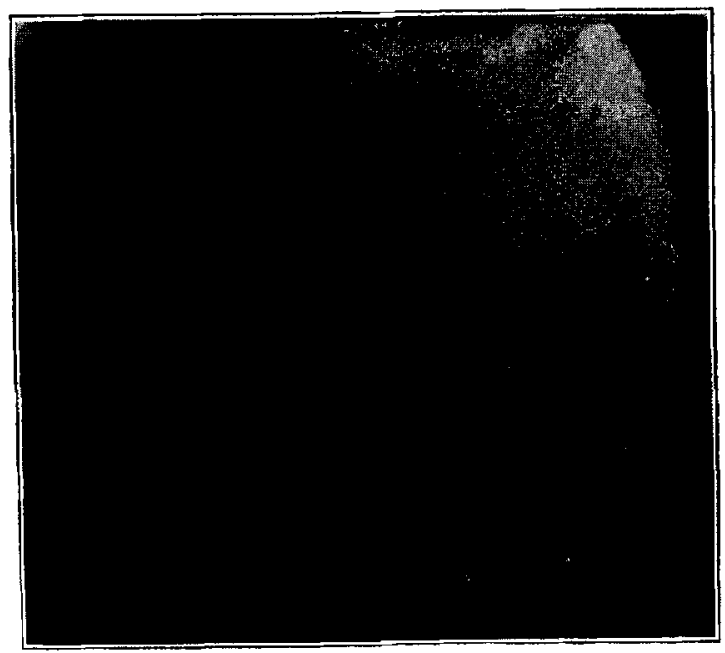

